\title{
Prevalence of peanut and tree nut allergy in the US determined by a random digit dial telephone survey
}

\author{
Scott H. Sicherer, MD, a Anne Muñoz-Furlong, BA, b A. Wesley Burks, MD,c and Hugh
}

A. Sampson, MDa New York, NY, Fairfax, Va, and Little Rock, Ark

\begin{abstract}
Background: Allergy to peanuts and tree nuts (TNs) is one of the leading causes of fatal and near-fatal food-induced allergic reactions. These allergies can be lifelong and appear to be increasing in prevalence. Despite the seriousness of these allergies, the prevalence of peanut and TN allergy in the general population is unknown.

Objective: We sought to determine the prevalence of peanut and TN allergy among the general population of the United States.
\end{abstract}

Methods: We used a nationwide, cross-sectional, random digit dial telephone survey with a standardized questionnaire. Results: A total of $\mathbf{4 3 7 4}$ households contacted by telephone participated (participation rate, $67 \%$ ), representing 12,032 individuals. Peanut or TN allergy was self-reported in 164 individuals $(1.4 \% ; 95 \%$ confidence interval [CI], $1.2 \%-1.6 \%)$ in 151 households $(3.5 \%$; $95 \%$ CI, $2.9 \%-4.0 \%)$. The prevalence of reported allergy in adults $(\mathbf{1 . 6 \%})$ was higher than that found in children under 18 years of age $(0.6 \%)$. In 131 individuals, details of the reactions were obtained. When applying criteria requiring reactions to be typical of IgE-mediated reactions (hives, angioedema, wheezing, throat tightness, vomiting, and diarrhea) within an hour of ingestion, $10 \%$ of these subjects were excluded. Among the remaining 118 subjects, allergic reactions involved 1 organ system (skin, respiratory, or gastrointestinal systems) in $\mathbf{5 0}$ subjects, 2 in $\mathbf{4 5}$ subjects, and all 3 in 23 subjects. Forty-five percent of these 118 respondents reported more than 5 lifetime reactions. Only $53 \%$ of these 118 subjects ever saw a physician for the allergic reaction, and only $7 \%$ had self-injectable epinephrine available at the time of the interview. The prevalence of peanut and TN allergy was adjusted by assuming that $10 \%$ of the remaining 33 subjects without a description of their reactions would also be excluded and correcting for a $7 \%$ false-positive rate for the survey

From aThe Elliot and Roslyn Jaffe Food Allergy Institute, Division of Allergy and Immunology, Department of Pediatrics, Mount Sinai School of Medicine, New York; bThe Food Allergy Network, Fairfax; and 'the Division of Immunology and Allergy, University of Arkansas for Medical Sciences, Arkansas Children's Hospital, Little Rock.

Supported by The Clarissa Sosin Allergy Research Foundation, Inc; The John and Susan Tomich Family Foundation; The Food Allergy Network; and The Jaffe Family Foundation.

Received for publication Dec 18, 1998; revised Jan 27, 1999; accepted for publication Jan 27, 1999,

Reprint requests: Scott H. Sicherer, MD, Division of Allergy/Immunology, Mount Sinai Hospital, Box 1198, One Gustave L. Levy Place, New York, New York 10029-6574.

Copyright $(\subset) 1999$ by Mosby, Inc.

$0091-6749 / 99 \$ 8.00+0 \quad \mathbf{1 / 1 / 9 7 4 0 0}$ instrument. A final "corrected" prevalence estimate of $\mathbf{1 . 1 \%}$ (95\% CI, 1.0\%-1.4\%) was obtained.

Conclusions: Peanut and/or TN allergy affects approximately $1.1 \%$ of the general population, or about 3 million Americans, representing a significant health concern. Despite the severity of reactions, about half of the subjects never sought an evaluation by a physician, and only a few had epinephrine available for emergency use. (J Allergy Clin Immunol 1999;103:559-62.)

Key words: Prevalence, peanut, tree nut, hypersensitivity, food allergy, anaphylaxis, telephone survey

There are several features of peanut (a legume) and tree nut (TN) (eg, walnut, almond, and cashew) allergy that have attracted recent attention in both the public press and in biomedical research. Although food-induced allergic reactions are the most common cause of anaphylaxis, ${ }^{1}$ peanuts and TNs are responsible for the majority of severe food-induced allergic reactions. ${ }^{2,3}$ In addition, patients allergic to peanuts and TNs rarely become tolerant of these foods ${ }^{4}$ and face a lifetime of avoidance. The ubiquitous nature of these foods, combined with the potential for cross-contamination in bakeries, restaurants, and processed food lines, makes avoidance difficult and accidental ingestion common. 4

Despite the seriousness of the problem, there have been no reports attempting to determine the prevalence of peanut and TN allergy in the general population. The only published report concerning the prevalence of peanut and TN allergy in unselected subjects was by Tariq et $\mathrm{al}^{5}$ who followed a birth cohort of 1218 children on the Isle of Wight. They found a $1.2 \%$ sensitization rate (on the basis of positive skin prick test responses) at 4 years of age, with 8 children $(0.5 \%)$ having experienced a clinical reaction. To estimate the prevalence of selfreported peanut and TN allergy among the general population of the United States, we conducted a random digit dial (RDD) telephone survey of 4374 participating households, representing 12,032 individuals.

\section{METHODS \\ Survey methods}

A nationwide, cross-sectional RDD telephone survey of households in the continental United States was performed by Innovative Medical Research, Inc (Towson, Maryland), using a questionnaire developed with the investigators that elicited primarily "yes/no" responses and was administered verbatim by using a computer- 
Abbreviations used

CI: Confidence interval

RDD: Random digit dialing

TN: Tree nut

assisted system. A straight, unweighted RDD method (random sampling of all telephone numbers in the continental US) was used, with a call list generated by the Genesys Sampling System (Fort Washington, $\mathrm{Pa}$ ). Up to 10 attempts were made to contact each telephone number. Nonresidential calls were excluded.

\section{Questionnaire contents, eligibility, surrogate rules, and data processing}

Verbal informed consent was obtained, and subject confidentiality was strictly preserved. Respondents were eligible if they were over 17 years of age, were living in the household, and understood the questions in English. Demographic information was obtained from all participating households. Respondents over age 17 years provided details about allergic reactions involving minors. Adults with $\mathrm{TN}$ or peanut allergy identified by a surrogate were included in the prevalence estimate, but details of reactions in adults were only included when the affected adult was personally interviewed. Up to 10 attempts were made to contact allergic adults identified by a surrogate. For individuals who reported peanut or TN allergy, further questions were administered to determine the details of the allergic reactions and the history of other allergic diseases.

Reactions were considered to be "convincing" if the organ systems affected and symptoms were typical of those involved in allergic reactions (skin system: hives and angioedema; respiratory system: trouble breathing, wheezing, and throat tightness; gastrointestinal system: vomiting and diarrhea) and occurred within 1 hour of ingestion. With this survey instrument and these criteria for a convincing acute allergic reaction, only $7 \%$ of 111 pediatric subjects did not have detectable serum nut-specific IgE antibodies. ${ }^{6}$

\section{RESULTS \\ Participation rate}

A total of 7036 households were contacted. Participation was refused by 2148 households for reasons including immediate refusal (1267), refusal after the concept of the survey was introduced (856), or denial of access to an eligible household member (25). An additional 455 households were ineligible as follows: age less than 18 years (166), unable to converse in English (227), hearing deficit or confusion (62), and incomplete interview (59). Thus 4374 of the 7036 households participated (67\%).

\section{Household characteristics, prevalence of self- reported peanut and TN allergy, and exclu- sion of subjects without convincing allergic reactions}

There were a total of 12,032 individuals in the 4374 households (mean, 2.75 persons per household). There were 1527 (35\%) males answering the questions among the 4363 in which the sex of the respondent was given. Peanut and/or TN allergy was reported in 151 households (3.5\%; $95 \%$ confidence interval $[\mathrm{CI}], 2.9 \%-4.0 \%$; no households with $>2$ individuals affected). There were
164 individuals with peanut and/or TN allergy, giving a prevalence rate of $1.4 \%$ (95\% CI, $1.2 \%-1.6 \%$ ). Table I shows the age distribution of the participants and those reporting peanut and TN allergy. Four adults reported both peanut and TN allergy. There was a higher prevalence of allergy in adults (1.6\%) than in children $(0.6 \%$; chi-square, $<0.0001, d f=17$ ).

Details about reactions were available for 131 of 164 (80\%) individuals (16 children and 115 adults). One child and 12 adults did not have convincing reactions. The child had only skin erythema from peanut, and the time from ingestion to reaction was not recalled. Among adults without convincing reactions, 7 reporting peanut allergy had no symptoms or unconvincing symptoms (sinusitis, migraine, and canker sores). Three adults reporting TN allergy had unrelated or unconvincing symptoms (possible diarrhea, isolated dizziness, and sores in mouth), and 2 adults reporting reactions to coconut were not considered TN reactors. Thus 118 of the 131 participants $(90 \%)$ in whom the details of the reaction were available met the criteria for a convincing allergic reaction.

\section{Corrected prevalence rate}

Assumptions were made to arrive at a "corrected" prevalence of peanut and TN allergy. Because $90 \%$ of subjects in whom details of the reaction were available fulfilled criteria for a convincing reaction, we assumed that $90 \%$ of the 33 individuals (29 individuals) with unknown reaction details also would have fulfilled the criteria. This results in 147 allergic individuals or a prevalence of $1.2 \%$ (95\% CI, $1.0 \%-1.4 \%$ ). Reducing the total by another $7 \%$ to reflect the potential false-positive rate of the survey, ${ }^{6}$ a corrected prevalence of $1.1 \%$ (95\% CI, $1.0 \%-1.4 \%$ ) was obtained.

\section{Clinical characteristics}

Detailed information about individuals with convincing reactions was available for 118 participants (33\% male). The number of individuals with reactions to each food was as follows: peanut, 58; walnut, 24; cashew, 8; Brazil nut, 8; almond, 7; pecan, 7; hazel nut, 3; Macadamia nut, 2; and unspecified mixed nuts, 6. Four adults reported both peanut and TN allergy, and 5 reported reactions to more than $1 \mathrm{TN}$.

The symptoms evoked during the first reaction in the 118 individuals included throat tightness (56\%), dyspnea $(43 \%)$, wheezing $(36 \%)$, angioedema $(51 \%)$, urticaria (42\%), vomiting (18\%), diarrhea (14\%), and loss of consciousness $(4 \%)$. One organ system was affected in 50 subjects (42\%), 2 in 45 subjects (38\%), and all 3 in 23 subjects (20\%). Reactions occurred shortly after exposure (mean, 5.7 minutes; median, 1.5 minutes), and all occurred within 30 minutes. Medications were used in 36 patients $(31 \%)$ on the first occurrence of a reaction and included the following: antihistamines (25 subjects), asthma medications (12 subjects), epinephrine (9 subjects), and corticosteroids ( 3 subjects). Multiple reactions were common: $55 \%$ reported 1 to 5 reactions, and $45 \%$ 
TABLE I. Age distribution of subjects with reported peanut and TN allergy

\begin{tabular}{lccccc}
\hline & $\begin{array}{c}\text { Total } \\
\text { population }\end{array}$ & $\begin{array}{c}\text { Allergy to TNs } \\
\text { and/or peanuts }\end{array}$ & $\begin{array}{c}\text { Allergy to } \\
\text { peanuts only }\end{array}$ & $\begin{array}{c}\text { Allergy to } \\
\text { TNs only }\end{array}$ & $\begin{array}{c}\text { Unspecified allergy to } \\
\text { peanut and/or TN }\end{array}$ \\
\hline $\begin{array}{l}\text { Adults } \\
\text { Children }\end{array}$ & 8049 & $131(1.6 \%)$ & $59(0.7 \%)$ & $59(0.7 \%)$ & $17(0.2 \%)$ \\
$\quad(<18$ y) & 2998 & $18(0.6 \%)$ & $12(0.4 \%)$ & $5(0.2 \%)$ & $1(0 \%)$ \\
Unspecified & 985 & $15(1.5 \%)$ & - & - & $15(1.5 \%)$ \\
Total & 12,032 & $164(1.4 \%)$ & $71(0.6 \%)$ & $64(0.5 \%)$ & $33(0.3 \%)$ \\
\hline
\end{tabular}

reported more than 5 lifetime reactions. Atopic diseases were also common $(51 \%$ had other food allergies, $35 \%$ had atopic dermatitis, 34\% had asthma, and 33\% had allergic rhinitis); $94 \%$ of subjects reported at least 1 of these atopic illnesses. Although $81 \%$ reported respiratory tract or multiple organ system involvement, only 62 of the $118(53 \%)$ were evaluated for the allergy by a physician. Fifteen of the $62(24 \%)$ that saw a physician $(90 \%$ had respiratory or multiple system reactions) were prescribed self-injectable epinephrine, and only 8 of these $15 \mathrm{had}$ the epinephrine available at the time of the interview.

\section{DISCUSSION}

Allergic reactions to peanuts and TNs are potentially life-threatening and were responsible for 5 of 7 near-fatal and 10 of 13 fatal food-induced anaphylactic reactions in 2 series. $^{2,3}$ In 2 case series of anaphylaxis treated in emergency departments, food allergy was the leading identifiable cause. ${ }^{7,8}$ Peanut or TN reactions accounted for $36 \%^{7}$ and $72 \% 8$ of these food-related episodes, respectively. Despite the potential severity of the allergy, the prevalence in the general population has been unknown. In this study, by using a RDD telephone survey of households in the US, $1.4 \%$ of individuals representing $3.5 \%$ of households reported peanut or TN allergy ( $1.6 \%$ of adults and $0.6 \%$ of children). After adjusting for subjects who did not provide a convincing history of an acute, allergic reaction and for a potential $7 \%$ falsepositive rate of the survey instrument, ${ }^{6}$ the corrected prevalence among the general US population is $1.1 \%$ (95\% CI, $1.0 \%-1.4 \%$ ).

There are a number of limitations in this study, some in regard to telephone surveys and others concerning the self-reported diagnosis of allergy. Telephone surveys may be biased in several respects. Overrepresentation of persons with a high socioeconomic status may occur because homes without telephones $(5.5 \%$ of homes according to the 1990 US Census $^{9}$ ) are excluded, ${ }^{10}$ and those with multiple voice lines are slightly more likely to be selected. Ethnic and racial biases may also occur. Because queries about race, ethnicity, income, and multiple telephone lines were not made in this study, the degree of such biases could not be determined. Telephone survey respondents are predominantly female, and a rate of $65 \%$ females, as found in this study, is not unusual. ${ }^{11}$ Despite the overrepresentation of women answering the survey, the demographic data on the households paralleled the 1990 US Census data. ${ }^{9}$ Although it is possible that the overrepresentation of females led to an underestimate of males with peanut and $\mathrm{TN}$ allergy, previous reports on patients with anaphylaxis have also shown a preponderance of adult females $(66 \%),{ }^{1}$ with more females affected as the age at reaction increased $(25 \%$ in children and $75 \%$ in adults over 40 years of age $\left.{ }^{8}\right)$.

There are also limitations of the survey instrument to identify true allergy. The "gold standard" for diagnosing food allergy is the double-blind, placebo-controlled oral food challenge. ${ }^{12}$ However, with a convincing history of a severe acute allergic reaction and positive skin or blood test results indicating specific IgE antibody, oral challenges may not be indicated. It was not practical in this study to challenge subjects taken from the general population. However, peanut and TN reactions are usually acute and severe, and therefore the false-positive rate of historical determination of allergy is generally low. In a study using this questionnaire instrument, $7 \%$ of 111 patients with convincing histories of acute reactions to peanuts or TNs did not have detectable peanut- or TNspecific serum IgE antibody, ${ }^{6}$ although this previous study was performed with children, and applicability to adult populations is only assumed. These patients may have false-positive results, they may have outgrown their allergy, or they may be a minority that has reactions with very low specific IgE antibody levels. Previous surveys have noted a false-positive rate of $13 \%$ (with a surrogate respondent for children using allergy skin prick tests to peanut to indicate sensitivity) to $14 \%$ (in adults). ${ }^{13,14}$

Several studies have addressed the characteristics of clinical reactions to peanuts and TNs, and the clinical characteristics of the reactions in this study were similar in terms of severity, associated atopic diseases, emergency medication use, and high frequency of accidental ingestions. ${ }^{2,6,14-16}$ However, 2 observations in this study were novel. First, only 4 subjects (all adults) reported allergy to both peanut and at least $1 \mathrm{TN}$. Previous studies in patients referred for allergy evaluations reported reactivity to $\mathrm{TN}$ in $20 \% 17$ and $34 \%$ of patients with peanut allergy. One explanation for the lower number of subjects with reactions to both peanut and TN in this study is that the respondents proffered information on either an allergy to peanut or TN without mentioning the other. This seems unlikely because subjects were prompted about both. Alternatively, allergy to both peanuts and TNs may 
not be as common in the general population compared with the rate reported in referral populations. The second novel finding was that these allergies were more common in adults than in children because the general prevalence of food allergy is generally greater in children $(7 \%)$ than in adults (1\% to $2 \%) .{ }^{18}$ Because peanut and $\mathrm{TN}$ allergies are usually not outgrown, ${ }^{4,19}$ there may be a greater representation among adults, a population that has accumulated affected individuals. The prevalence of peanutinduced $(0.4 \%)$ and $\mathrm{TN}$-induced $(0.2 \%)$ allergic reactions among children in this study was similar to that reported in a birth cohort of 1218 children in the Isle of Wight evaluated at age 4 years $(0.5 \%$ for peanut and $0.2 \%$ for $\mathrm{TN}) .^{5}$

This study also revealed important issues about treatment of these allergies. Only half of the patients consulted a physician about their allergy. Also worrisome was the fact that epinephrine was apparently underprescribed, a finding previously reported. ${ }^{20,21}$ Despite the fact that $90 \%$ of the patients seeing a physician had reactions involving the respiratory tract or multiple organ systems, only $24 \%$ were prescribed epinephrine. Only $53 \%$ of those subjects prescribed epinephrine had it available at the time of the survey.

This telephone survey indicates that $1.4 \%$ of the general US population report peanut/TN allergy. In applying conservative criteria to correct for unconvincing histories and false-positive determinations, at least $1.1 \%$ of the general population, or about 3 million Americans, has experienced acute allergic reactions to peanuts or TNs. Most of these individuals experienced multiple and severe reactions. Despite the seriousness of the allergy, relatively few sought medical attention, and for those who did, self-injectable epinephrine was underprescribed. Implications for the identification, education, and treatment of patients and increased research efforts are evident.

\section{REFERENCES}

1. Yocum MW, Khan DA. Assessment of patients who have experienced anaphylaxis: a 3-year survey. Mayo Clin Proc 1994;69:16-23.
2. Sampson HA, Mendelson LM, Rosen JP. Fatal and near-fatal anaphylactic reactions to food in children and adolescents. $\mathrm{N}$ Engl J Med 1992;327:380-4.

3. Yunginger JW, Sweeney KG, Sturner WQ, Giannandra LA, Teigland JD, Bray M, et al. Fatal food-induced anaphylaxis. JAMA 1988;260:1450-2.

4. Bock SA, Atkins FM. The natural history of peanut allergy. J Allergy Clin Immunol 1989;83:900-4.

5. Tariq SM, Stevens M, Matthews S, Ridout S, Twiselton R, Hide DW. Cohort study of peanut and tree nut sensitisation by age of 4 years. BMJ 1996;313:514-7.

6. Sicherer SH, Burks AW, Sampson HA. Clinical features of acute allergic reactions to peanut and tree nuts in children. Pediatrics 1998;102:e6.

7. Kemp SF, Lockey RF, Wolf BL, Lieberman P. Anaphylaxis: a review of 266 cases. Arch Intern Med 1995;155:1749-54.

8. Pumphrey RSH, Stanworth SJ. The clinical spectrum of anaphylaxis in north-west England. Clin Exp Allergy 1996;26:1364-70.

9. US Census Bureau. 1990 Census data, STF3C1. Available from: http://venus.census.gov/cdrom/lookup. Accessed 1998.

10. Waksberg J. Sampling methods for random digit dialing. J Am Stat Assoc 1978;73:40-6

11. Stewart WF, Lipton RB, Liberman J. Variation in migraine prevalence by race. Neurology 1996;47:52-9.

12. Bock SA, Sampson HA, Atkins FM, Zeiger RS, Lehrer S, Sachs M, et al. Double-blind, placebo-controlled food challenge (DBPCFC) as an office procedure: a manual. J Allergy Clin Immunol 1988;82:986-97.

13. Hourihane JO'B, Dean TP, Warner JO. Peanut allergy in relation to heredity, maternal diet, and other atopic diseases: results of a questionnaire survey, skin prick testing, and food challenges. BMJ 1996;313:51821.

14. Hourihane JO'B, Kilburn SA, Dean P, Warner JO. Clinical characteristics of peanut allergy. Clin Exp Allergy 1997;27:634-9.

15. Fries JH. Peanuts: allergic and other untoward reactions. Ann Allergy 1982;48:220-6.

16. Furlong TJ, Sicherer SH, Munoz-Furlong A, Burks AW, Sampson HA. Establishment of a Registry for allergic reactions to peanut [abstract]. J Allergy Clin Immunol 1998;101:S104.

17. Ewan P. Clinical study of peanut and nut allergy in 62 consecutive patients: new features and associations. BMJ 1996;312:1074-8.

18. Sampson HA. Food Allergy. JAMA 1997;278:1888-94.

19. Hourihane JO, Roberts SA, Warner JO. Resolution of peanut allergy: case-control study. BMJ 1998;316:1271-5.

20. Klein JS, Yocum MW. Underreporting of anaphylaxis in a community emergency room. J Allergy Clin Immunol 1995;95:637-8.

21. Hutcheson PS, Slavin RG. Lack of preventive measures given to patients with stinging insect anaphylaxis in hospital emergency rooms. Ann Allergy 1990;64:306-7. 